\title{
Mathematical aspects of the Heisenberg uncertainty principle within local fractional Fourier analysis
}

\author{
Xiao-Jun Yang ${ }^{1,2,3^{*}}$, Dumitru Baleanu ${ }^{4,5,6}$ and José António Tenreiro Machado ${ }^{7}$
}

\author{
"Correspondence: \\ dyangxiaojun@163.com \\ 1 Department of Mathematics and \\ Mechanics, China University of \\ Mining and Technology, Xuzhou \\ Campus, Xuzhou, Jiangsu 221008, \\ China \\ ${ }^{2}$ Institute of Software Science, \\ Zhengzhou Normal University, \\ Zhengzhou, 450044, China \\ Full list of author information is \\ available at the end of the article
}

\begin{abstract}
In this paper, we discuss the mathematical aspects of the Heisenberg uncertainty principle within local fractional Fourier analysis. The Schrödinger equation and Heisenberg uncertainty principles are structured within local fractional operators.
\end{abstract}

Keywords: Heisenberg uncertainty principle; local fractional Fourier operator; Schrödinger equation; fractal time-space

\section{Introduction}

As it is known, the fractal curves $[1,2]$ are everywhere continuous but nowhere differentiable; therefore, we cannot use the classical calculus to describe the motions in Cantor time-space [3-10]. The theory of local fractional calculus [11-20], started to be considered as one of the useful tools to handle the fractal and continuously non-differentiable functions. This formalism was applied in describing physical phenomena such as continuum mechanics [21], elasticity [20-22], quantum mechanics [23, 24], heat-diffusion and wave phenomena [25-30], and other branches of applied mathematics [31-33] and nonlinear dynamics $[34,35]$.

The fractional Heisenberg uncertainty principle and the fractional Schrödinger equation based on fractional Fourier analysis were proposed [36-48]. Local fractional Fourier analysis [49], which is a generalization of the Fourier analysis in fractal space, has played an important role in handling non-differentiable functions. The theory of local fractional Fourier analysis is structured in a generalized Hilbert space (fractal space), and some results were obtained [26, 49-53]. Also, its applications were investigated in quantum mechanics [23], differentials equations [26, 28] and signals [51].

The main purpose of this paper is to present the mathematical aspects of the Heisenberg uncertainty principle within local fractional Fourier analysis and to structure a local fractional version of the Schrödinger equation.

The manuscript is structured as follows. In Section 2, the preliminary results for the local fractional calculus are investigated. The theory of local fractional Fourier analysis is introduced in Section 3. The Heisenberg uncertainty principle in local fractional Fourier analysis is studied in Section 4. Application of quantum mechanics in fractal space is considered in Section 5. Finally, the conclusions are presented in Section 6.

C) 2013 Yang et al.; licensee Springer. This is an Open Access article distributed under the terms of the Creative Commons Attribution License (http://creativecommons.org/licenses/by/2.0), which permits unrestricted use, distribution, and reproduction in any medium, provided the original work is properly cited. 


\section{Mathematical tools}

\subsection{Local fractional continuity of functions}

Definition 1 [18-20, 27-30] If there is

$$
\left|f(x)-f\left(x_{0}\right)\right|<\varepsilon^{\alpha}
$$

with $\left|x-x_{0}\right|<\delta$, for $\varepsilon, \delta>0$ and $\varepsilon, \delta \in \mathbb{R}$. Now $f(x)$ is called a local fractional continuous at $x=x_{0}$, denoted by $\lim _{x \rightarrow x_{0}} f(x)=f\left(x_{0}\right)$. Then $f(x)$ is called local fractional continuous on the interval $(a, b)$, denoted by

$$
f(x) \in C_{\alpha}(a, b) .
$$

The function $f(x)$ is said to be local fractional continuous at $x_{0}$ from the right if $f\left(x_{0}\right)$ is defined, and

$$
\lim _{x \rightarrow x_{0}^{+}} f(x)=f\left(x_{0}^{+}\right) .
$$

The function $f(x)$ is said to be local fractional continuous at $x_{0}$ from the left if $f\left(x_{0}\right)$ is defined, and

$$
\lim _{x \rightarrow x_{0}^{-}} f(x)=f\left(x_{0}^{-}\right) .
$$

Suppose that $\lim _{x \rightarrow x_{0}^{+}} f(x)=f\left(x_{0}^{+}\right), \lim _{x \rightarrow x_{0}} f(x)=f\left(x_{0}^{-}\right)$and $f\left(x_{0}^{+}\right)=f\left(x_{0}^{-}\right)$, then we have the following relation:

$$
\lim _{x \rightarrow x_{0}^{+}} f(x)=\lim _{x \rightarrow x_{0}^{-}} f(x)=\lim _{x \rightarrow x_{0}} f(x) .
$$

For other results of theory of local fractional continuity of functions, see [18-20, 27-30].

\subsection{Local fractional derivative and integration}

Definition $2[18-20,27-30]$ Setting $f(x) \in C_{\alpha}(a, b)$, a local fractional derivative of $f(x)$ of order $\alpha$ at $x=x_{0}$ is defined by

$$
f^{(\alpha)}\left(x_{0}\right)=\left.\frac{d^{\alpha} f(x)}{d x^{\alpha}}\right|_{x=x_{0}}=\lim _{x \rightarrow x_{0}} \frac{\Delta^{\alpha}\left(f(x)-f\left(x_{0}\right)\right)}{\left(x-x_{0}\right)^{\alpha}}
$$

where $\Delta^{\alpha}\left(f(x)-f\left(x_{0}\right)\right) \cong \Gamma(1+\alpha) \Delta\left(f(x)-f\left(x_{0}\right)\right)$ with a gamma function $\Gamma(1+\alpha)$.

Definition $3[18-20,27-30]$ Setting $f(x) \in C_{\alpha}(a, b)$, a local fractional integral of $f(x)$ of order $\alpha$ in the interval $[a, b]$ is defined as

$$
{ }_{a} I_{b}^{(\alpha)} f(x)=\frac{1}{\Gamma(1+\alpha)} \int_{a}^{b} f(t)(d t)^{\alpha}=\frac{1}{\Gamma(1+\alpha)} \lim _{\Delta t \rightarrow 0} \sum_{j=0}^{j=N-1} f\left(t_{j}\right)\left(\Delta t_{j}\right)^{\alpha}
$$

where $\Delta t_{j}=t_{j+1}-t_{j}, \Delta t=\max \left\{\Delta t_{1}, \Delta t_{2}, \Delta t_{j}, \ldots\right\}$ and $\left[t_{j}, t_{j+1}\right], j=0, \ldots, N-1, t_{0}=a, t_{N}=b$, is a partition of the interval $[a, b]$. 
Their fractal geometrical explanation of local fractional derivative and integration can be seen in $[22,26,50-52]$.

If $f(x) \in C_{\alpha}[a, b]$, then we have $[18,19]$

$$
\left|{ }_{a} I_{b}^{(\alpha)} f(x)\right| \leq{ }_{a} I_{b}^{(\alpha)}|f(x)|
$$

with $b-a>0$.

Lemma $1[18,19]$

$$
\left[{ }_{-\infty} I_{\infty}^{(\alpha)} f(x) g(x)\right]^{2} \leq\left[{ }_{-\infty} I_{\infty}^{(\alpha)}|g(x)|^{2}\right]\left[{ }_{-\infty} I_{\infty}^{(\alpha)}|g(x)|^{2} g(x)\right]
$$

Proof See $[18,19]$.

\section{Theory of local fractional Fourier analysis}

In this section, we investigate local fractional Fourier analysis [49-53], which is a generalized Fourier analysis in fractal space. Here we discuss the local fractional Fourier series, the Fourier transform and the generalized Fourier transform in fractal space. We start with a local fractional Fourier series.

\subsection{Local fractional Fourier series}

Definition $4[18,19,49-52]$ The local fractional trigonometric Fourier series of $f(t)$ is given by

$$
f(t)=a_{0}+\sum_{i=1}^{\infty} a_{k} \sin _{\alpha}\left(k^{\alpha} \omega_{0}^{\alpha} t^{\alpha}\right)+\sum_{i=1}^{\infty} b_{k} \cos _{\alpha}\left(k^{\alpha} \omega_{0}^{\alpha} t^{\alpha}\right) .
$$

Then the local fractional Fourier coefficients can be computed by

$$
\left\{\begin{array}{l}
a_{0}=\frac{1}{T^{\alpha}} \int_{0}^{T} f(t)(d t)^{\alpha} \\
a_{k}=\left(\frac{2}{T}\right)^{\alpha} \int_{0}^{T} f(t) \sin _{\alpha}\left(k^{\alpha} \omega_{0}^{\alpha} t^{\alpha}\right)(d t)^{\alpha}, \\
b_{k}=\left(\frac{2}{T}\right)^{\alpha} \int_{0}^{T} f(t) \cos _{\alpha}\left(k^{\alpha} \omega_{0}^{\alpha} t^{\alpha}\right)(d t)^{\alpha}
\end{array}\right.
$$

The Mittag-Leffler functions expression of the local fractional Fourier series is described by $[18,19,49-52]$

$$
f(x)=\sum_{k=-\infty}^{\infty} C_{k} E_{\alpha}\left(\frac{\pi^{\alpha} i^{\alpha}(k x)^{\alpha}}{l^{\alpha}}\right)
$$

where the local fractional Fourier coefficients are

$$
C_{k}=\frac{1}{(2 l)^{\alpha}} \int_{-l}^{l} f(x) E_{\alpha}\left(\frac{-\pi^{\alpha} i^{\alpha}(k x)^{\alpha}}{l^{\alpha}}\right)(d x)^{\alpha} \quad \text { with } k \in \mathbb{Z}
$$

The above is generalized to calculate the local fractional Fourier series. 


\subsection{The Fourier transform in fractal space}

Definition $5[18,19,49-53]$ Suppose that $f(x) \in C_{\alpha}(-\infty, \infty)$, the Fourier transform in fractal space, denoted by $F_{\alpha}\{f(x)\} \equiv f_{\omega}^{F, \alpha}(\omega)$, is written in the form

$$
F_{\alpha}\{f(x)\}=\frac{1}{\Gamma(1+\alpha)} \int_{-\infty}^{\infty} E_{\alpha}\left(-i^{\alpha} \omega^{\alpha} x^{\alpha}\right) f(x)(d x)^{\alpha},
$$

where the latter converges.

Definition $6[18,19,49-53]$ If $F_{\alpha}\{f(x)\} \equiv f_{\omega}^{F, \alpha}(\omega)$, its inversion formula is written in the form

$$
f(x)=\frac{1}{(2 \pi)^{\alpha}} \int_{-\infty}^{\infty} E_{\alpha}\left(i^{\alpha} \omega^{\alpha} x^{\alpha}\right) f_{\omega}^{F, \alpha}(\omega)(d \omega)^{\alpha}, \quad x>0 .
$$

\subsection{The generalized Fourier transform in fractal space}

Definition $7[18,19]$ The generalized Fourier transform in fractal space is written in the form

$$
F_{\alpha}\{f(x)\}=\frac{1}{\Gamma(1+\alpha)} \int_{-\infty}^{\infty} f(x) E_{\alpha}\left(-i^{\alpha} h_{0} x^{\alpha} \omega^{\alpha}\right)(d x)^{\alpha},
$$

where $h_{0}=\frac{(2 \pi)^{\alpha}}{\Gamma(1+\alpha)}$ with $0<\alpha \leq 1$.

Definition $8[18,19]$ The inverse formula of the generalized Fourier transform in fractal space is written in the form $[18,19]$

$$
f(x)=\frac{1}{\Gamma(1+\alpha)} \int_{-\infty}^{\infty} f_{\omega}^{F, \alpha}(\omega) E_{\alpha}\left(i^{\alpha} h_{0} x^{\alpha} \omega^{\alpha}\right)(d \omega)^{\alpha},
$$

where $h_{0}=\frac{(2 \pi)^{\alpha}}{\Gamma(1+\alpha)}$ with $0<\alpha \leq 1$.

\subsection{Some useful results}

The following formula is valid $[18,19]$.

Theorem $1[18,19]$

$$
F_{\alpha}\left\{f^{(\alpha)}(x)\right\}=i^{\alpha} h_{0} \omega^{\alpha} F_{\alpha}\{f(x)\} .
$$

Proof See $[18,19]$.

Theorem $2[18,19]$ If $F_{\alpha}\{f(x)\}=f_{\omega}^{F, \alpha}(\omega)$, then we have

$$
\int_{-\infty}^{\infty}|f(x)|^{2}(d x)^{\alpha}=\int_{-\infty}^{\infty}\left|f_{\omega}^{F, \alpha}(\omega)\right|^{2}(d \omega)^{\alpha} .
$$

Proof See $[18,19]$.

Theorem $3[18,19]$ If $F_{\alpha}\{f(x)\}=f_{\omega}^{F, \alpha}(\omega)$, then we have

$$
\int_{-\infty}^{\infty} f(x) \overline{g(x)}(d x)^{\alpha}=\int_{-\infty}^{\infty} f_{\omega}^{F, \alpha}(\omega) \overline{g_{\omega}^{F, \alpha}(\omega)}(d \omega)^{\alpha} .
$$


Proof See $[18,19]$.

\section{Heisenberg uncertainty principles in local fractional Fourier analysis}

Theorem 4 Suppose that $f \in L_{2, \alpha}[\Re], F_{\alpha}\{f(x)\}=f_{\omega}^{F, \alpha}(\omega)$, then we have

$$
\frac{\Gamma^{2}(1+\alpha)}{4 h_{0}^{2}} \leq\left[\frac{\frac{1}{\Gamma(1+\alpha)} \int_{-\infty}^{\infty}\left[f(x) x^{\alpha}\right]^{2}(d x)^{\alpha}}{\frac{1}{\Gamma(1+\alpha)} \int_{-\infty}^{\infty}|f(x)|^{2}(d x)^{\alpha}}\right] \cdot\left[\frac{\frac{1}{\Gamma(1+\alpha)} \int_{-\infty}^{\infty}\left[f_{\omega}^{F, \alpha}(\omega) \omega^{\alpha}\right]^{2}(d \omega)^{\alpha}}{\frac{1}{\Gamma(1+\alpha)} \int_{-\infty}^{\infty}|f(x)|^{2}(d x)^{\alpha}}\right],
$$

with equality only if $f(x)$ is almost everywhere equal to a constant multiple of

$$
C_{0} E_{\alpha}\left(\frac{-x^{2 \alpha}}{K}\right)
$$

with $K>0$ and a constant $C_{0}$.

Proof Considering the equality

$$
\frac{1}{\Gamma(1+\alpha)} \int_{-\infty}^{\infty}\left[f_{\omega}^{F, \alpha}(\omega) h_{0} \omega^{\alpha}\right]^{2}(d \omega)^{\alpha}=\frac{1}{\Gamma(1+\alpha)} \int_{-\infty}^{\infty}\left[f^{(\alpha)}(x)\right]^{2}(d x)^{\alpha},
$$

we have

$$
\begin{aligned}
& {\left[\frac{1}{\Gamma(1+\alpha)} \int_{-\infty}^{\infty}\left[f(x) x^{\alpha}\right]^{2}(d x)^{\alpha}\right]\left[\frac{1}{\Gamma(1+\alpha)} \int_{-\infty}^{\infty}\left[f_{\omega}^{F, \alpha}(\omega) h_{0} \omega^{\alpha}\right]^{2}(d x)^{\alpha}\right]} \\
& \quad=\left[\frac{1}{\Gamma(1+\alpha)} \int_{-\infty}^{\infty}\left[f(x) x^{\alpha}\right]^{2}(d x)^{\alpha}\right]\left[\frac{1}{\Gamma(1+\alpha)} \int_{-\infty}^{\infty}\left[f^{(\alpha)}(x)\right]^{2}(d x)^{\alpha}\right] \\
& \quad \geq \frac{1}{\Gamma(1+\alpha)} \int_{-\infty}^{\infty}\left|\overline{f(x)} f^{(\alpha)}(x) x^{\alpha}\right|^{2}(d x)^{\alpha} \\
& \quad \geq \mid \frac{1}{\Gamma(1+\alpha)} \int_{-\infty}^{\infty} \overline{\left.f(x) f^{(\alpha)}(x) x^{\alpha}(d x)^{\alpha}\right|^{2} .}
\end{aligned}
$$

When $\frac{f(x) x^{\alpha}}{K}=f^{(\alpha)}(x)$, then we have $f(x)=C_{0} E_{\alpha}\left(-\frac{x^{2 \alpha}}{K}\right)$ with a constant $C_{0}$.

Since

$$
\left(|f(x)|^{2}\right)^{(\alpha)}=(f(x) \overline{f(x)})^{(\alpha)}=f^{(\alpha)}(x) \overline{f(x)}+f(x) \overline{f^{(\alpha)}(x)}
$$

and

$$
\left|\frac{1}{\Gamma(1+\alpha)} \int_{-\infty}^{\infty} f^{(\alpha)}(x) \overline{f(x)} x^{\alpha}(d x)^{\alpha}\right|=\left|\frac{1}{\Gamma(1+\alpha)} \int_{-\infty}^{\infty} f(x) \overline{f^{(\alpha)}(x)} x^{\alpha}(d x)^{\alpha}\right|,
$$

we have

$$
\begin{aligned}
& \frac{1}{\Gamma(1+\alpha)} \int_{-\infty}^{\infty}\left(|f(x)|^{2}\right)^{(\alpha)} x^{\alpha}(d x)^{\alpha} \\
& \quad=\left[\left(|f(x)|^{2}\right) x^{\alpha}\right]_{-\infty}^{\infty}-\int_{-\infty}^{\infty}|f(x)|^{2}(d x)^{\alpha} \\
& \quad=-\int_{-\infty}^{\infty}|f(x)|^{2}(d x)^{\alpha},
\end{aligned}
$$

when $\left(|f(x)|^{2}\right) x^{\alpha} \rightarrow 0, x \rightarrow \infty$. 
Hence, there is

$$
\begin{aligned}
& \left.\left|\int_{-\infty}^{\infty}\right| f(x)\right|^{2}(d x)^{\alpha} \mid \\
& \quad=\left|\frac{1}{\Gamma(1+\alpha)} \int_{-\infty}^{\infty}\left(|f(x)|^{2}\right)^{(\alpha)} x^{\alpha}(d x)^{\alpha}\right| \\
& \quad=\left|\frac{1}{\Gamma(1+\alpha)} \int_{-\infty}^{\infty} f^{(\alpha)}(x) \overline{f(x)} x^{\alpha}(d x)^{\alpha}+\frac{1}{\Gamma(1+\alpha)} \int_{-\infty}^{\infty} x^{\alpha} f(x) \overline{f^{(\alpha)}(x)}(d x)^{\alpha}\right| \\
& \quad \leq \frac{2}{\Gamma(1+\alpha)}\left|\int_{-\infty}^{\infty} f^{(\alpha)}(x) \overline{f(x)} x^{\alpha}(d x)^{\alpha}\right|
\end{aligned}
$$

such that

$$
\begin{aligned}
& \left|\frac{1}{\Gamma(1+\alpha)} \int_{-\infty}^{\infty}\left(|f(x)|^{2}\right)^{(\alpha)} x^{\alpha}(d x)^{\alpha}\right|^{2} \\
& \quad=\left.\left.\left|\int_{-\infty}^{\infty}\right| f(x)\right|^{2}(d x)^{\alpha}\right|^{2} \\
& \quad=\left.\left.\Gamma^{2}(1+\alpha)\left|\frac{1}{\Gamma(1+\alpha)} \int_{-\infty}^{\infty}\right| f(x)\right|^{2}(d x)^{\alpha}\right|^{2} \\
& \quad \leq 4\left(\frac{1}{\Gamma(1+\alpha)} \int_{-\infty}^{\infty} f^{(\alpha)}(x) \overline{f(x)} x^{\alpha}(d x)^{\alpha}\right)^{2} \\
& \quad \leq 4\left(\frac{1}{\Gamma(1+\alpha)} \int_{-\infty}^{\infty}\left|\overline{f(x)} f^{(\alpha)}(x) x^{\alpha}\right|(d x)^{\alpha}\right)^{2} \\
& \quad \leq 4\left[\frac{1}{\Gamma(1+\alpha)} \int_{-\infty}^{\infty}\left[f(x) x^{\alpha}\right]^{2}(d x)^{\alpha}\right]\left[\frac{1}{\Gamma(1+\alpha)} \int_{-\infty}^{\infty}\left[f_{\omega}^{F, \alpha}(\omega) h_{0} \omega^{\alpha}\right]^{2}(d \omega)^{\alpha}\right] .
\end{aligned}
$$

Therefore, we deduce to

$$
\begin{aligned}
& \left.\left.\Gamma^{2}(1+\alpha)\left|\frac{1}{\Gamma(1+\alpha)} \int_{-\infty}^{\infty}\right| f(x)\right|^{2}(d x)^{\alpha}\right|^{2} \\
& \quad \leq 4\left[\frac{1}{\Gamma(1+\alpha)} \int_{-\infty}^{\infty}\left[f(x) x^{\alpha}\right]^{2}(d x)^{\alpha}\right]\left[\frac{1}{\Gamma(1+\alpha)} \int_{-\infty}^{\infty}\left[f_{\omega}^{F, \alpha}(\omega) h_{0} \omega^{\alpha}\right]^{2}(d \omega)^{\alpha}\right] \\
& \quad=4 h_{0}^{2}\left[\frac{1}{\Gamma(1+\alpha)} \int_{-\infty}^{\infty}\left[f(x) x^{\alpha}\right]^{2}(d x)^{\alpha}\right]\left[\frac{1}{\Gamma(1+\alpha)} \int_{-\infty}^{\infty}\left[f_{\omega}^{F, \alpha}(\omega) \omega^{\alpha}\right]^{2}(d \omega)^{\alpha}\right] .
\end{aligned}
$$

Hence, this result is obtained.

As a direct result, we have two equivalent forms as follows.

Theorem 5 Suppose that $f \in L_{2, \alpha}[\Re]$ and $f^{(\alpha)}(x)=\frac{d^{\alpha} f(x)}{d x^{\alpha}}$, then we have

$$
\frac{\Gamma^{2}(1+\alpha)}{4} \leq\left[\frac{\frac{1}{\Gamma(1+\alpha)} \int_{-\infty}^{\infty}\left[f(x) x^{\alpha}\right]^{2}(d x)^{\alpha}}{\frac{1}{\Gamma(1+\alpha)} \int_{-\infty}^{\infty}|f(x)|^{2}(d x)^{\alpha}}\right] \cdot\left[\frac{\frac{1}{\Gamma(1+\alpha)} \int_{-\infty}^{\infty}\left[f^{(\alpha)}(x)\right]^{2}(d x)^{\alpha}}{\frac{1}{\Gamma(1+\alpha)} \int_{-\infty}^{\infty}|f(x)|^{2}(d x)^{\alpha}}\right]
$$

with equality only if $f(x)$ is almost everywhere equal to a constant multiple of $C_{0} E_{\alpha}\left(\frac{-x^{2 \alpha}}{K}\right)$, with $K>0$ and a constant $C_{0}$. 
Proof Applying Theorem 4, we have

$$
\frac{1}{\Gamma(1+\alpha)} \int_{-\infty}^{\infty}\left[f^{(\alpha)}(x)\right]^{2}(d x)^{\alpha}=\frac{1}{\Gamma(1+\alpha)} \int_{-\infty}^{\infty}\left[f_{\omega}^{F, \alpha}(\omega) h_{0} \omega^{\alpha}\right]^{2}(d \omega)^{\alpha}
$$

such that

$$
\frac{\Gamma^{2}(1+\alpha)}{4} \leq\left[\frac{\frac{1}{\Gamma(1+\alpha)} \int_{-\infty}^{\infty}\left[f(x) x^{\alpha}\right]^{2}(d x)^{\alpha}}{\frac{1}{\Gamma(1+\alpha)} \int_{-\infty}^{\infty}|f(x)|^{2}(d x)^{\alpha}}\right] \cdot\left[\frac{\frac{1}{\Gamma(1+\alpha)} \int_{-\infty}^{\infty}\left[f^{(\alpha)}(x)\right]^{2}(d x)^{\alpha}}{\frac{1}{\Gamma(1+\alpha)} \int_{-\infty}^{\infty}|f(x)|^{2}(d x)^{\alpha}}\right]
$$

Hence, Theorem 5 is obtained.

The above results $[37,38]$ are different from the results in fractional Fourier transform $[36,37]$ based on the fractional calculus theory.

\section{The mathematical aspect of fractal quantum mechanics}

\subsection{Local fractional Schrödinger equation}

We structure the non-differential phase of a fractal plane wave as a complex phase factor using the relations

$$
\begin{aligned}
\psi_{\alpha} & =A E_{\alpha}\left(i^{\alpha}\left(\bar{k}^{\alpha} \bar{r}^{\alpha}-\omega^{\alpha} t^{\alpha}\right)\right) \\
& =A E_{\alpha}\left(\frac{i^{\alpha}}{h_{\alpha}}\left(\bar{P}_{\alpha} \bar{r}^{\alpha}-E_{\alpha} t^{\alpha}\right)\right),
\end{aligned}
$$

where the Planck-Einstein and De Broglie relations are in fractal space

$$
\left\{\begin{array}{l}
E_{\alpha}=h_{\alpha} \omega^{\alpha} \\
P_{\alpha}=h_{\alpha} k^{\alpha}
\end{array}\right.
$$

We can realize the local fractional partial derivative with respect to fractal space

$$
\begin{aligned}
\nabla^{\alpha} \psi_{\alpha} & =\frac{i^{\alpha}}{h_{\alpha}} P_{\alpha} A E_{\alpha}\left(\frac{i^{\alpha}}{h_{\alpha}}\left(\bar{P}_{\alpha} \bar{r}^{\alpha}-E_{\alpha} t^{\alpha}\right)\right) \\
& =\frac{i^{\alpha}}{h_{\alpha}} P_{\alpha} \psi_{\alpha}
\end{aligned}
$$

and fractal time

$$
\begin{aligned}
\frac{\partial^{\alpha} \psi_{\alpha}}{\partial t^{\alpha}} & =-\frac{i^{\alpha}}{h_{\alpha}} E_{\alpha} A E_{\alpha}\left(\frac{i^{\alpha}}{h_{\alpha}}\left(\bar{P}_{\alpha} \bar{r}^{\alpha}-E_{\alpha} t^{\alpha}\right)\right) \\
& =-\frac{i^{\alpha}}{h_{\alpha}} E_{\alpha} \psi_{\alpha},
\end{aligned}
$$

where $\nabla^{\alpha}=\frac{\partial^{\alpha}}{\partial x^{\alpha}} i^{\alpha}+\frac{\partial^{\alpha}}{\partial y^{\alpha}} j^{\alpha}+\frac{\partial^{\alpha}}{\partial z^{\alpha}} k^{\alpha}[26]$ with $\bar{r}^{\alpha}=x^{\alpha} i^{\alpha}+y^{\alpha} j^{\alpha}+z^{\alpha} k^{\alpha}[26]$.

From (5.3) we have

$$
-i^{\alpha} h_{\alpha} \nabla^{\alpha} \psi_{\alpha}=P_{\alpha} \psi_{\alpha}
$$


such that

$$
-\frac{h_{\alpha}^{2}}{2 m} \nabla^{2 \alpha} \psi_{\alpha}=\frac{1}{2 m} \bar{P}_{\alpha} \cdot \bar{P}_{\alpha} \psi_{\alpha}
$$

where $\nabla^{2 \alpha}=\frac{\partial^{2 \alpha}}{\partial x^{2 \alpha}}+\frac{\partial^{2 \alpha}}{\partial y^{2 \alpha}}+\frac{\partial^{2 \alpha}}{\partial z^{2 \alpha}}$ with $\bar{r}^{\alpha}=x^{\alpha} i^{\alpha}+y^{\alpha} j^{\alpha}+z^{\alpha} k^{\alpha}$.

From (5.4) we have

$$
i^{\alpha} h_{\alpha} \frac{\partial^{\alpha} \psi_{\alpha}}{\partial t^{\alpha}}=E_{\alpha} \psi_{\alpha} .
$$

We have the energy equation

$$
\begin{aligned}
E_{\alpha} & =\frac{1}{2 m} \bar{P}_{\alpha} \cdot \bar{P}_{\alpha}+V_{\alpha} \\
& =H_{\alpha}
\end{aligned}
$$

such that

$$
E_{\alpha} \psi_{\alpha}=H_{\alpha} \psi_{\alpha}
$$

and

$$
E_{\alpha} \psi_{\alpha}=-\frac{h_{\alpha}^{2}}{2 m} \nabla^{2 \alpha} \psi_{\alpha}+V_{\alpha} \psi_{\alpha}
$$

where $H_{\alpha}$ is the local fractional Hamiltonian in fractal mechanics.

Hence, we have that

$$
i^{\alpha} h_{\alpha} \frac{\partial^{\alpha} \psi_{\alpha}}{\partial t^{\alpha}}=-\frac{h_{\alpha}^{2}}{2 m} \nabla^{2 \alpha} \psi_{\alpha}+V_{\alpha} \psi_{\alpha}
$$

Therefore, we can deduce that the local fractional energy operator is

$$
\bar{E}_{\alpha}=i^{\alpha} h_{\alpha} \frac{\partial^{\alpha}}{\partial t^{\alpha}}
$$

and that the local fractional momentum operator is

$$
\bar{P}_{\alpha}=i^{\alpha} h_{\alpha} \nabla^{\alpha} .
$$

Therefore, we get the local fractional Schrödinger equation in the form of local fractional energy and momentum operators

$$
H_{\alpha} \psi_{\alpha}=\frac{1}{2 m} \bar{P}_{\alpha} \cdot \bar{P}_{\alpha} \psi_{\alpha}+V_{\alpha} \psi_{\alpha},
$$

where the local fractional Hamiltonian is

$$
H_{\alpha}=\frac{1}{2 m} \bar{P}_{\alpha} \cdot \bar{P}_{\alpha}+V_{\alpha}
$$


We also deduce that the general time-independent local fractional Schrödinger equation is written in the form

$$
i^{\alpha} h_{\alpha} \frac{\partial^{\alpha} \psi_{\alpha}}{\partial t^{\alpha}}=H_{\alpha} \psi_{\alpha}
$$

which is related to the following equation:

$$
\frac{\partial^{\alpha} S_{\alpha}\left(q_{i}, t\right)}{\partial t^{\alpha}}=H_{\alpha}\left(q_{i}, \frac{\partial^{\alpha} S_{\alpha}}{\partial q_{i}^{\alpha}}, t\right)
$$

where $S_{\alpha}$ is non-differential action, $H_{\alpha}$ is the local fractional Hamiltonian function, and $q_{i}^{\alpha}(i=1,2,3)$ are generalized fractal coordinates.

\subsection{Solutions of the local fractional Schrödinger equation}

\subsubsection{General solutions of the local fractional Schrödinger equation}

The general solution of the local fractional Schrödinger equation can be seen in the following. For discrete $k$, the sum is a superposition of fractal plane waves:

$$
\begin{aligned}
\psi_{\alpha}(r, t) & =\sum_{n=1}^{\infty} A_{n} E_{\alpha}\left(i^{\alpha}\left(k_{n}^{\alpha} r^{\alpha}-\omega_{n}^{\alpha} t^{\alpha}\right)\right) \\
& =\sum_{n=1}^{\infty} A_{n} E_{\alpha}\left(\frac{i^{\alpha}}{h_{\alpha}}\left(\bar{P}_{\alpha} \bar{r}^{\alpha}-E_{\alpha} t^{\alpha}\right)\right) \\
& =\sum_{n=1}^{\infty} A_{n} E_{\alpha}\left(\frac{i^{\alpha}}{h_{\alpha}}\left(\bar{P}_{\alpha} \bar{r}^{\alpha}-\frac{\bar{P}_{\alpha}^{2}}{2 m} t^{\alpha}\right)\right)
\end{aligned}
$$

and

$$
E_{\alpha}=\frac{P_{\alpha}^{2}}{2 m}
$$

If we consider $\bar{P}_{\alpha}=p_{x \alpha} i^{\alpha}+p_{y \alpha} j^{\alpha}+p_{z \alpha} k^{\alpha} \equiv p_{x \alpha} i^{\alpha}$ and $\bar{r}^{\alpha}=x^{\alpha} i^{\alpha}+z^{\alpha} j^{\alpha}+z^{\alpha} k^{\alpha}$, we have fractal plane waves:

$$
\begin{aligned}
\psi_{\alpha}(x, t) & =\psi_{\alpha}\left(P_{x \alpha}, t\right) \\
& =\sum_{n=1}^{\infty} A_{n} E_{\alpha}\left(\frac{i^{\alpha}}{h_{\alpha}}\left(p_{x \alpha} x^{\alpha}-\frac{p_{x \alpha}^{2}}{2 m} t^{\alpha}\right)\right) .
\end{aligned}
$$

\subsubsection{Fractal complex wave functions}

The meaning of this description can be seen in the following. Similar to the classical wave mechanics, we prepare $N$ atoms independently, in the same state, so that when each of them is measured, they are described by the same wave function. Then the result of a position measurement is described as the fractal probability density, and we wish it is not the same for all. The set of impacts is distributed in space with the probability density

$$
\phi_{\alpha}(\bar{P}(r), t)=\left|\psi_{\alpha}(r, t)\right|^{2} .
$$


In view of (5.20), we have

$$
\phi_{\alpha}(x, t)=\left|\psi_{\alpha}(x, t)\right|^{2}
$$

The set of $N$ measurements is characterized by an expectation value $\langle r\rangle_{\alpha}$ and a root mean square dispersion $(\Delta r)^{\alpha}$,

$$
\langle r\rangle_{\alpha}=\frac{1}{\Gamma^{3}(1+\alpha)} \int_{-\infty}^{\infty} r^{\alpha}\left|\psi_{\alpha}(r, t)\right|^{2}(d r)^{\alpha} .
$$

Similarly, the square of the dispersion $(\Delta r)^{2 \alpha}$ is defined by

$$
\begin{aligned}
(\Delta r)^{2 \alpha} & =\left\langle x^{2}\right\rangle_{\alpha}-\left(\langle x\rangle_{\alpha}\right)^{2} \\
& =\left\langle\left(x^{\alpha}-\langle x\rangle_{\alpha}\right)^{2}\right\rangle_{\alpha} \\
& =\frac{1}{\Gamma^{3}(1+\alpha)} \int_{-\infty}^{\infty}\left(r^{\alpha}-\langle r\rangle_{\alpha}\right)^{2}\left|\psi_{\alpha}(r, t)\right|^{2}(d r)^{\alpha} .
\end{aligned}
$$

If the physical interpretation of a particle in fractal space is that the probability

$$
d P(r)=\frac{1}{\Gamma^{3}(1+\alpha)}\left|\psi_{\alpha}(r, t)\right|^{2}(d r)^{3 \alpha},
$$

the integral of this quantity over all fractal space is

$$
\begin{aligned}
P(r) & =\frac{1}{\Gamma^{3}(1+\alpha)} \int_{-\infty}^{\infty}\left|\psi_{\alpha}(r, t)\right|^{2}(d r)^{3 \alpha} \\
& =1 .
\end{aligned}
$$

For (5.18) we have

$$
\begin{aligned}
\psi_{\alpha}(r, t) & =\sum_{n=1}^{\infty} A_{n} E_{\alpha}\left(i^{\alpha}\left(k_{n}^{\alpha} r^{\alpha}-\omega_{n}^{\alpha} t^{\alpha}\right)\right) \\
& =\sum_{n=1}^{\infty} A_{n} E_{\alpha}\left(\frac{i^{\alpha}}{h_{\alpha}}\left(\bar{P}_{\alpha} \bar{r}^{\alpha}-E_{\alpha} t^{\alpha}\right)\right) \\
& =\sum_{n=1}^{\infty} A_{n} E_{\alpha}\left(\frac{i^{\alpha}}{h_{\alpha}}\left(\bar{P}_{\alpha} \bar{r}^{\alpha}-\frac{\bar{P}_{\alpha}^{2}}{2 m} t^{\alpha}\right)\right)
\end{aligned}
$$

such that

$$
1=\frac{1}{\Gamma^{3}(1+\alpha)} \int_{-\infty}^{\infty}\left|\psi_{\alpha}(r, t)\right|^{2}(d r)^{3 \alpha} .
$$

5.2.3 Probabilistic interpretation of fractal complex wave function of one variable In (5.22), we have

$$
\phi_{\alpha}(x, t)=\left|\psi_{\alpha}(x, t)\right|^{2}
$$


and

$$
P(x)=1
$$

such that

$$
\begin{aligned}
& \frac{1}{\Gamma(1+\alpha)} \int_{-\infty}^{\infty}\left|\psi_{\alpha}(x, t)\right|^{2}(d x)^{\alpha} \\
& \quad=\frac{1}{\Gamma(1+\alpha)} \int_{-L}^{L}\left|A E_{\alpha}\left(\frac{i^{\alpha}}{h_{\alpha}}\left(p_{x \alpha} x^{\alpha}-\frac{p_{x \alpha}^{2}}{2 m} t^{\alpha}\right)\right)\right|^{2}(d x)^{\alpha} \\
& \quad=\frac{2 A^{2} L^{\alpha}}{\Gamma(1+\alpha)} \\
& \quad=1
\end{aligned}
$$

where

$$
\psi_{\alpha}(x, t)= \begin{cases}A E_{\alpha}\left(\frac{i^{\alpha}}{h_{\alpha}}\left(p_{x \alpha} x^{\alpha}-\frac{p_{x \alpha}^{2}}{2 m} t^{\alpha}\right)\right), & x \in L, \\ 0, & x \notin L .\end{cases}
$$

We have an expectation value $\langle x\rangle_{\alpha}$ and a root mean square dispersion $(\Delta x)^{\alpha}$,

$$
\langle x\rangle_{\alpha}=\frac{1}{\Gamma(1+\alpha)} \int_{-\infty}^{\infty} x^{\alpha}\left|\psi_{\alpha}(x, t)\right|^{2}(d x)^{\alpha}
$$

and

$$
\begin{aligned}
(\Delta x)^{2 \alpha} & =\left\langle x^{2}\right\rangle_{\alpha}-\left(\langle x\rangle_{\alpha}\right)^{2} \\
& =\left\langle\left(x^{\alpha}-\langle x\rangle_{\alpha}\right)^{2}\right\rangle_{\alpha} \\
& =\frac{1}{\Gamma(1+\alpha)} \int_{-\infty}^{\infty}\left(x^{\alpha}-\langle x\rangle_{\alpha}\right)^{2}\left|\psi_{\alpha}(x, t)\right|^{2}(d x)^{\alpha} .
\end{aligned}
$$

For a given fractal mechanical operator $A$, we have an expectation value $\langle A\rangle_{\alpha}$ and a root mean square dispersion $(\Delta A)^{\alpha}$,

$$
\langle A\rangle_{\alpha}=\frac{1}{\Gamma(1+\alpha)} \int_{-\infty}^{\infty} A\left|\psi_{\alpha}(x, t)\right|^{2}(d x)^{\alpha}
$$

and

$$
\begin{aligned}
(\Delta A)^{2 \alpha} & =\left\langle\left(A-\langle A\rangle_{\alpha}\right)^{2}\right\rangle_{\alpha} \\
& =\left\langle A^{2}\right\rangle_{\alpha}-\left(\langle A\rangle_{\alpha}\right)^{2} \\
& =\frac{1}{\Gamma(1+\alpha)} \int_{-\infty}^{\infty}\left(A-\langle A\rangle_{\alpha}\right)^{2}\left|\psi_{\alpha}(x, t)\right|^{2}(d x)^{\alpha} .
\end{aligned}
$$




\subsection{The Heisenberg uncertainty principle in fractal quantum mechanics}

Suppose that

$$
\frac{1}{\Gamma(1+\alpha)} \int_{-\infty}^{\infty}\left|\psi_{\alpha}(x, t)\right|^{2}(d x)^{\alpha}=1,
$$

we have a fractal positional operator expectation value

$$
\langle x\rangle_{\alpha}=\frac{1}{\Gamma(1+\alpha)} \int_{-\infty}^{\infty} i^{\alpha} x^{\alpha}\left|\psi_{\alpha}(x, t)\right|^{2}(d x)^{\alpha}=0
$$

and a root mean square dispersion of positional operator

$$
(\Delta x)^{2 \alpha}=\frac{1}{\Gamma(1+\alpha)} \int_{-\infty}^{\infty} i^{2 \alpha} x^{2 \alpha}\left|\psi_{\alpha}(x, t)\right|^{2}(d x)^{\alpha} .
$$

Similar to the fractal positional operator, we have a fractal momentum operator expectation value

$$
\left\langle P_{x}\right\rangle_{\alpha}=\left\langle i^{\alpha} h_{\alpha} \frac{\partial^{\alpha}}{\partial x^{\alpha}}\right\rangle_{\alpha}=\frac{1}{\Gamma(1+\alpha)} \int_{-\infty}^{\infty} i^{\alpha} h_{\alpha} \frac{\partial^{\alpha}}{\partial x^{\alpha}}\left|\psi_{\alpha}(x, t)\right|^{2}(d x)^{\alpha}=0
$$

and a root mean square dispersion of positional operator

$$
\left(\Delta P_{x}\right)^{2 \alpha}=\frac{1}{\Gamma(1+\alpha)} \int_{-\infty}^{\infty} i^{2 \alpha} h_{\alpha}^{2} \frac{\partial^{2 \alpha}}{\partial x^{2 \alpha}}\left|\psi_{\alpha}(x, t)\right|^{2}(d x)^{\alpha} .
$$

Considering

$$
\begin{aligned}
& (\Delta x)^{2 \alpha}=\frac{1}{\Gamma(1+\alpha)} \int_{-\infty}^{\infty} i^{2 \alpha} x^{2 \alpha}\left|\psi_{\alpha}(x, t)\right|^{2}(d x)^{\alpha}, \\
& \left(\Delta P_{x}\right)^{2 \alpha}=\frac{1}{\Gamma(1+\alpha)} \int_{-\infty}^{\infty} i^{2 \alpha} h_{\alpha}^{2} \frac{\partial^{2 \alpha}}{\partial x^{2 \alpha}}\left|\psi_{\alpha}(x, t)\right|^{2}(d x)^{\alpha},
\end{aligned}
$$

and

$$
\frac{1}{\Gamma(1+\alpha)} \int_{-\infty}^{\infty}\left|\psi_{\alpha}(x, t)\right|^{2}(d x)^{\alpha}=1,
$$

by using Theorem 5 , we have that

$$
\frac{\Gamma^{2}(1+\alpha)}{4} \leq\left[\frac{1}{\Gamma(1+\alpha)} \int_{-\infty}^{\infty} x^{2 \alpha}\left|\psi_{\alpha}(x, t)\right|^{2}(d x)^{\alpha}\right]\left[\frac{1}{\Gamma(1+\alpha)} \int_{-\infty}^{\infty} \frac{\partial^{2 \alpha}}{\partial x^{2 \alpha}}\left|\psi_{\alpha}(x, t)\right|^{2}(d x)^{\alpha}\right]
$$

such that

$$
\frac{\Gamma^{2}(1+\alpha)}{4} \leq(\Delta x)^{2 \alpha} \frac{\left(\Delta P_{x}\right)^{2 \alpha}}{h_{\alpha}^{2}} .
$$

Hence, we have that

$$
\frac{\Gamma^{2}(1+\alpha) h_{\alpha}^{2}}{4} \leq(\Delta x)^{2 \alpha}\left(\Delta P_{x}\right)^{2 \alpha}
$$


such that

$$
\frac{\Gamma(1+\alpha) h_{\alpha}}{2} \leq(\Delta x)^{\alpha}\left(\Delta P_{x}\right)^{\alpha},
$$

where

$$
(\Delta x)^{\alpha}=\sqrt{(\Delta x)^{2 \alpha}}=\sqrt{\frac{1}{\Gamma(1+\alpha)} \int_{-\infty}^{\infty} i^{2 \alpha} x^{2 \alpha}\left|\psi_{\alpha}(x, t)\right|^{2}(d x)^{\alpha}}
$$

and

$$
\left(\Delta P_{x}\right)^{\alpha}=\sqrt{\left(\Delta P_{x}\right)^{2 \alpha}}=\sqrt{\frac{1}{\Gamma(1+\alpha)} \int_{-\infty}^{\infty} i^{2 \alpha} h_{\alpha}^{2} \frac{\partial^{2 \alpha}}{\partial x^{2 \alpha}}\left|\psi_{\alpha}(x, t)\right|^{2}(d x)^{\alpha}} .
$$

Suppose that

$$
h_{\alpha}=\left(\frac{h}{2 \pi}\right)^{\alpha},
$$

then we have

$$
\frac{\Gamma(1+\alpha)\left(\frac{h}{2 \pi}\right)^{\alpha}}{2} \leq(\Delta x)^{\alpha}\left(\Delta P_{x}\right)^{\alpha}
$$

and

$$
i^{\alpha}\left(\frac{h}{2 \pi}\right)^{\alpha} \frac{\partial^{\alpha} \psi_{\alpha}}{\partial t^{\alpha}}=-\frac{\left(\frac{h}{2 \pi}\right)^{2 \alpha}}{2 m} \nabla^{2 \alpha} \psi_{\alpha}+V_{\alpha} \psi_{\alpha},
$$

where $\nabla^{2 \alpha}=\frac{\partial^{2 \alpha}}{\partial x^{2 \alpha}}+\frac{\partial^{2 \alpha}}{\partial y^{2 \alpha}}+\frac{\partial^{2 \alpha}}{\partial z^{2 \alpha}}[24]$.

The above equation (5.50) differs from the results presented in [36, 37]. Also, Eq. (5.51) is different from the ones reported in [38-40, 54, 55].

Below we define the local fractional energy operator

$$
\bar{E}_{\alpha}=i^{\alpha}\left(\frac{h}{2 \pi}\right)^{\alpha} \frac{\partial^{\alpha}}{\partial t^{\alpha}}
$$

and the local fractional momentum operator

$$
\bar{P}_{\alpha}=i^{\alpha}\left(\frac{h}{2 \pi}\right)^{\alpha} \nabla^{\alpha},
$$

where $\nabla^{\alpha}=\frac{\partial^{\alpha}}{\partial x^{\alpha}} i^{\alpha}+\frac{\partial^{\alpha}}{\partial y^{\alpha}} j^{\alpha}+\frac{\partial^{\alpha}}{\partial z^{\alpha}} k^{\alpha}[26]$.

Thus, we get the Planck-Einstein and de Broglie relations are in fractal space as

$$
\left\{\begin{array}{l}
E_{\alpha}=\left(\frac{h}{2 \pi}\right)^{\alpha} \omega^{\alpha}, \\
P_{\alpha}=\left(\frac{h}{2 \pi}\right)^{\alpha} k^{\alpha},
\end{array}\right.
$$

where $h$ is Planck's constant. 


\section{Conclusions}

In this manuscript, the uncertainty principle in local fractional Fourier analysis is suggested. Since the local fractional calculus can be applied to deal with the non-differentiable functions defined on any fractional space, the local fractional Fourier transform is important to deal with fractal signal functions. The results on uncertainty principles could play an important role in time-frequency analysis in fractal space. From Eq. (A.7) we conclude that there is a semi-group property for the Mittag-Leffler function on fractal sets. Meanwhile, uncertainty principles derived from local fractional Fourier analysis are classical uncertainty principles in the case of $\alpha=1$. We reported the structure the local fractional Schrödinger equation derived from Planck-Einstein and de Broglie relations in fractal time space.

\section{Appendix}

We have $[13,20]$

$$
\gamma^{\alpha}[F, a, b]+\gamma^{\alpha}[F, b, c]=\gamma^{\alpha}[F, a, c]
$$

such that

$$
S_{F}^{\alpha}(y)-S_{F}^{\alpha}(x)=\gamma^{\alpha}[F, x, y]=\frac{(y-x)^{\alpha}}{\Gamma(1+\alpha)},
$$

where $S_{F}^{\alpha}(y)$ is a fractal integral staircase function. We have the relation [18-20]

$$
H^{\alpha}(F \cap(\gamma, 0))=-\gamma^{\alpha}
$$

such that

$$
S_{F}^{\alpha}(y)-S_{F}^{\alpha}(x)=\gamma^{\alpha}[F, x, y]=H^{\alpha}(F \cap(x, y))=\frac{(y-x)^{\alpha}}{\Gamma(1+\alpha)} .
$$

Inversely we obtain

$$
S_{F}^{\alpha}(x)-S_{F}^{\alpha}(y)=\gamma^{\alpha}[F, y, x]=H^{\alpha}(F \cap(y, x))=-\frac{(y-x)^{\alpha}}{\Gamma(1+\alpha)} .
$$

Hence, both $S_{F}^{\alpha}(x)=\frac{x^{\alpha}}{\Gamma(1+\alpha)}$ and $S_{F}^{\alpha}(y)=\frac{y^{\alpha}}{\Gamma(1+\alpha)}$ are seen in [20, 21].

In view of Eq. (A.4), we easily obtain that

$$
E_{\alpha}\left(i^{\alpha} x^{\alpha}\right)=\cos _{\alpha} x^{\alpha}+i^{\alpha} \sin _{\alpha} x^{\alpha}
$$

and

$$
E_{\alpha}\left(x^{\alpha}+y^{\alpha}\right)=E_{\alpha}\left((x+y)^{\alpha}\right)=E_{\alpha}\left(x^{\alpha}\right) E_{\alpha}\left(y^{\alpha}\right)
$$

where $E_{\alpha}\left(x^{\alpha}\right)=\sum_{k=0}^{\infty} \frac{x^{\alpha k}}{\Gamma(1+k \alpha)}, \sin _{\alpha} x^{\alpha}=\sum_{k=0}^{\infty} \frac{(-1)^{k} x^{(2 k+1) \alpha}}{\Gamma[1+(2 k+1) \alpha]}, \cos _{\alpha} x^{\alpha}=\sum_{k=0}^{\infty} \frac{(-1)^{k} x^{2 \alpha k}}{\Gamma(1+2 \alpha k)}$ and $i^{\alpha}$ is a fractal unit of imaginary number [18-20, 53]. 


\section{Competing interests}

The authors declare that they have no competing interests.

\section{Authors' contributions}

Authors contributed equally in writing this article. Authors read and approved the final manuscript.

\section{Author details}

1 Department of Mathematics and Mechanics, China University of Mining and Technology, Xuzhou Campus, Xuzhou, Jiangsu 221008, China. ${ }^{2}$ Institute of Software Science, Zhengzhou Normal University, Zhengzhou, 450044, China. ${ }^{3}$ Institute of Applied Mathematics, Qujing Normal University, Qujing, 655011, China. ${ }^{4}$ Department of Mathematics and Computer Sciences, Faculty of Arts and Sciences, Cankaya University, Ankara, 06530, Turkey. ${ }^{5}$ Institute of Space Sciences, Magurele, Bucharest, Romania. ${ }^{6}$ Department of Chemical and Materials Engineering, Faculty of Engineering, King Abdulaziz University, P.O. Box 80204, Jeddah, 21589, Saudi Arabia. ${ }^{7}$ Department of Electrical Engineering, Institute of Engineering of Polytechnic of Porto, Rua Dr. Antonio Bernardino de Almeida, 431, Porto, 4200-072, Portugal.

\section{Acknowledgements}

Dedicated to Professor Hari M Srivastava.

The authors would like to thank to the referees for their very useful comments and remarks.

\section{Received: 29 January 2013 Accepted: 2 May 2013 Published: 20 May 2013}

\section{References}

1. Mandelbrot, BB: The Fractal Geometry of Nature. Freeman, New York (1982)

2. Falconer, KJ: Fractal Geometry-Mathematical Foundations and Application. Wiley, New York (1997)

3. Zeilinger, A, Svozil, K: Measuring the dimension of space-time. Phys. Rev. Lett. 54(24), 2553-2555 (1985)

4. Nottale, L: Fractals and the quantum theory of space-time. Int. J. Mod. Phys. A 4(19), 5047-5117 (1989)

5. Saleh, AA: On the dimension of micro space-time. Chaos Solitons Fractals 7(6), 873-875 (1996)

6. Maziashvili, M: Space-time uncertainty relation and operational definition of dimension. (2007) arXiv:0709.0898

7. Caruso, F, Oguri, V: The cosmic microwave background spectrum and an upper limit for fractal space dimensionality. Astrophys. J. 694(1), 151-156 (2009)

8. Calcagni, G: Geometry and field theory in multi-fractional spacetime. J. High Energy Phys. 65(1), 1-65 (2012)

9. Kong, HY, He, JH: A novel friction law. Therm. Sci. 16(5), 1529-1533 (2012)

10. Kong, HY, He, JH: The fractal harmonic law and its application to swimming suit. Therm. Sci. 16(5), 1467-1471 (2012)

11. Kolwankar, KM, Gangal, AD: Fractional differentiability of nowhere differentiable functions and dimensions. Chaos 6(4), 505-513 (1996)

12. Jumarie, G: Table of some basic fractional calculus formulae derived from a modified Riemann-Liouville derivative for non-differentiable functions. Appl. Math. Lett. 22, 378-385 (2009)

13. Parvate, A, Gangal, AD: Calculus on fractal subsets of real line - I: formulation. Fractals 17(1), 53-81 (2009)

14. Chen, W: Time-space fabric underlying anomalous diffusion. Chaos Solitons Fractals 28, $923-929$ (2006)

15. Adda, FB, Cresson, J: About non-differentiable functions. J. Math. Anal. Appl. 263, 721-737 (2001)

16. Balankin, AS, Elizarraraz, BE: Map of fluid flow in fractal porous medium into fractal continuum flow. Phys. Rev. E 85(5), 056314 (2012)

17. He, JH: A new fractal derivation. Therm. Sci. 15, 145-147 (2011)

18. Yang, XJ: Local fractional integral transforms. Prog. Nonlinear Sci. 4, 1-225 (2011)

19. Yang, XJ: Local Fractional Functional Analysis and Its Applications. Asian Academic Publisher, Hong Kong (2011)

20. Yang, XJ: Advanced Local Fractional Calculus and Its Applications. World Science Publisher, New York (2012)

21. Carpinteri, A, Chiaia, B, Cornetti, P: Static-kinematic duality and the principle of virtual work in the mechanics of fractal media. Comput. Methods Appl. Mech. Eng. 191, 3-19 (2001)

22. Carpinteri, A, Cornetti, P: A fractional calculus approach to the description of stress and strain localization in fractal media. Chaos Solitons Fractals 13(1), 85-94 (2002)

23. Yang, XJ: The zero-mass renormalization group differential equations and limit cycles in non-smooth initial value problems. Prespacetime J. 3(9), 913-923 (2012)

24. Kolwankar, KM, Gangal, AD: Local fractional Fokker-Planck equation. Phys. Rev. Lett. 80, 214-217 (1998)

25. Wu, GC, Wu, KT: Variational approach for fractional diffusion-wave equations on Cantor sets. Chin. Phys. Lett. 29(6), 060505 (2012)

26. Zhong, WP, Gao, F, Shen, XM: Applications of Yang-Fourier transform to local fractional equations with local fractional derivative and local fractional integral. Adv. Mater. Res. 461, 306-310 (2012)

27. Yang, XJ, Baleanu, D: Fractal heat conduction problem solved by local fractional variation iteration method. Therm. Sci. (2012). doi:10.2298/TSCI121124216Y

28. Hu, MS, Agarwal, RP, Yang, XJ: Local fractional Fourier series with application to wave equation in fractal vibrating string. Abstr. Appl. Anal. 2012, Article ID 567401 (2012)

29. Yang, XJ, Baleanu, D, Zhong, WP: Approximation solution to diffusion equation on Cantor time-space. Proc. Rom. Acad., Ser. A: Math. Phys. Tech. Sci. Inf. Sci. (2013, in press)

30. Hu, MS, Baleanu, D, Yang, XJ: One-phase problems for discontinuous heat transfer in fractal media. Math. Probl. Eng. 2013, Article ID 358473 (2013)

31. Babakhani, A, Gejji, VD: On calculus of local fractional derivatives. J. Math. Anal. Appl. 270(1), 66-79 (2002)

32. Chen, Y, Yan, Y, Zhang, K: On the local fractional derivative. J. Math. Anal. Appl. 362, 17-33 (2010)

33. Kim, TS: Differentiability of fractal curves. Commun. Korean Math. Soc. 20(4), 827-835 (2005)

34. Parvate, A, Gangal, AD: Fractal differential equations and fractal-time dynamical systems. Pramana J. Phys. 64(3), 389-409 (2005)

35. Yang, XJ, Liao, MK, Wang, JN: A novel approach to processing fractal dynamical systems using the Yang-Fourie transforms. Adv. Electr. Eng. Syst. 1(3), 135-139 (2012) 
36. Namias, V: The fractional order Fourier transform and its application to quantum mechanics. IMA J. Appl. Math. 25(3), 241-265 (1980)

37. Mustard, D: Uncertainty principles invariant under the fractional Fourier transform. J. Aust. Math. Soc. 33(2), 180-191 (1991)

38. Bhatti, M: Fractional Schrödinger wave equation and fractional uncertainty principle. Int. J. Contemp. Math. Sci. 19(2), 943-950 (2007)

39. Laskin, N: Fractional quantum mechanics. Phys. Rev. E 62, 3135-3145 (2000)

40. Laskin, N: Fractional quantum mechanics and Lévy path integrals. Phys. Lett. A 268, 298-305 (2000)

41. Laskin, N: Fractional Schrödinger equation. Phys. Rev. E 66, 056108 (2002)

42. Muslih, SI, Agrawal, OP, Baleanu, D: A fractional Schrödinger equation and its solution. Int. J. Theor. Phys. 49(8), 1746-1752 (2010)

43. Adda, FB, Cresson, J: Quantum derivatives and the Schrödinger equation. Chaos Solitons Fractals 19, 1323-1334 (2004)

44. Tofighi, A: Probability structure of time fractional Schrödinger equation. Acta Phys. Pol. 116(2), 114-118 (2009)

45. Naber, M: Time fractional Schrödinger equation. J. Math. Phys. 45(8), 3325-3339 (2004)

46. Dong, JP, Xu, MY: Some solutions to the space fractional Schrödinger equation using momentum representation method. J. Math. Phys. 48, 072105 (2007)

47. Rozmej, P, Bandrowski, B: On fractional Schrödinger equation. Comput. Methods Sci. Technol. 16(2), 191-194 (2010)

48. Iomin, A: Fractional-time Schrödinger equation: fractional dynamics on a comb. Chaos Solitons Fractals 44, 348-352 (2011)

49. Liao, MK, Yang, XJ, Yan, Q: A new viewpoint to Fourier analysis in fractal space. In: Anastassiou, GA, Duman, O (eds.) Advances in Applied Mathematics and Approximation Theory, Chapter 26, pp. 399-411. Springer, New York (2013)

50. Guo, Y: Local fractional Z transform in fractal space. Adv. Digit. Multimed. 1(2), 96-102 (2012)

51. Yang, XJ, Liao, MK, Chen, JW: A novel approach to processing fractal signals using the Yang-Fourier transforms. Proc. Eng. 29, 2950-2954 (2012)

52. Yang, XJ: Theory and applications of local fractional Fourier analysis. Adv. Mech. Eng. Appl. 1(4), $70-85$ (2012)

53. He, JH: Asymptotic methods for solitary solutions and compactons. Abstr. Appl. Anal. 2012, Article ID 916793 (2012)

54. $\mathrm{He}, \mathrm{JH}$ : Frontier of modern textile engineering and short remarks on some topics in physics. Int. J. Nonlinear Sci. Numer. Simul. 11(7), 555-563 (2010)

55. Yang, CD: Trajectory interpretation of the uncertainty principle in 1D systems using complex Bohmian mechanics. Phys. Lett. A 372(41), 6240-6253 (2008)

doi:10.1186/1687-2770-2013-131

Cite this article as: Yang et al.: Mathematical aspects of the Heisenberg uncertainty principle within local fractional

Fourier analysis. Boundary Value Problems 2013 2013:131.

\section{Submit your manuscript to a SpringerOpen ${ }^{\circ}$ journal and benefit from:}

- Convenient online submission

Rigorous peer review

- Immediate publication on acceptance

- Open access: articles freely available online

- High visibility within the field

- Retaining the copyright to your article 